\title{
Augmenting the Driver's View with Peripheral Information on a Windshield Display
}

\author{
Renate Häuslschmid ${ }^{1}$, Sven Osterwald ${ }^{2}$, Marcus Lang ${ }^{3}$, Andreas Butz ${ }^{4}$ \\ 1,2,3,4 University of Munich (LMU), ${ }^{1,2,3}$ IAV GmbH, Munich \\ ${ }^{1}$ renate.haeuslschmid@iav.de, ${ }^{2}$ osterwald@cip.ifi.lmu.de, ${ }^{3}$ marcus@martinlang.com, ${ }^{4}$ butz@ifi.lmu.de
}

\begin{abstract}
Windshield displays (WSDs) are information displays covering the entire windshield. Current WSD test setups place information at different distances, but always within the driver's foveal field of view. We built two WSD test setups, which present information not only at various distances within the driver's visual focus, but also in the peripheral field of view. Then we evaluated the display of information in the periphery on both WSD setups in a user study. While making sure the participants would look at the peripheral information, we measured the display's impact on driving performance. Subjects were also asked about their driving experience with the windshield displays and their preference among the two setups.
\end{abstract}

\section{Author Keywords}

In-vehicle information system; visual focus; periphery; widget board; driving performance; user experience.

\section{ACM Classification Keywords}

\section{H.1.2; H.5.2.}

\section{General Terms}

Human Factors; Performance.

\section{INTRODUCTION}

Windshield displays (WSDs) are the big siblings of HeadUp Displays (HUDs). Both place information within the drivers' view through the windshield, which can provide a substantial safety benefit over so-called Head-Down Displays such as the instrument panel. While drivers need to move their head down to see information in those standard in-vehicle displays, WSDs and HUDs let them keep their heads up and the road situation in view. Drivers can stay aware of the road situation much easier and are less strained when changing focus between the virtual image and the road scene. The distant image enables a faster eye accommodation, and in turn a faster perception of the virtual image, resulting in shorter reaction times $[7,8,10]$.

Permission to make digital or hard copies of all or part of this work for personal or classroom use is granted without fee provided that copies are not made or distributed for profit or commercial advantage and that copies bear this notice and the full citation on the first page. Copyrights for components of this work owned by others than ACM must be honored. Abstracting with credit is permitted. To copy otherwise, or republish, to post on servers or to redistribute to lists, requires prior specific permission and/or a fee. Request permissions from Permissions@acm.org. IUI'15, March 29 - April 01, 2015, Atlanta, GA, USA

Copyright is held by the owner/author(s). Publication rights licensed to ACM. ACM 978-1-4503-3306-1/15/03 _\$15.00

http://dx.doi.org/10.1145/2678025.2701393

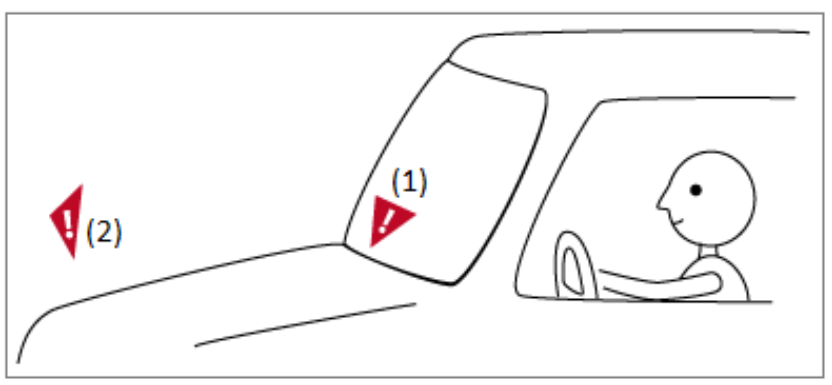

Figure 1: Information on a windshield display at different distances to the driver: (1) on the windshield, (2) in front of it

The term windshield display already implies the difference to HUDs: WSDs use large parts of the windshield area as a display pane. HUDs, on the contrary, usually cover only 3 to 6 inches [2]. The small display area strongly limits the content to driving- and safety-relevant information. Such displays could provide many opportunities as the dashboard area is increasingly crowded [22]. Functions currently located on the central information display could be relocated to the WSD, which offers a large space for information supporting the primary (driving) task, but also secondary and tertiary tasks. WSD applications could support safety (e.g., by displaying the distance to a leading vehicle, warnings or by highlighting hazards), navigation (e.g., through a route markup), or influence driver behavior (e.g., by an ambient display of fuel consumption).

Information could either be placed in the central region currently covered by HUDs, or in other parts of the driver's foveal and peripheral field of view (FoV). The foveal area is also the most relevant area for driving. Overlaying this space with a display can have disadvantages [6], as information in this area can occlude parts of the driving scene or even hazards. Using the space in the periphery has a much lower risk of occluding the driving scene. By periphery in this context we mean the area surrounding the foveal FoV, which corresponds to the borders of the WSD if the driver looks straight at the road scene. As the driving scene is spread out horizontally, the top and bottom areas of the windshield are good candidate areas for displaying information, which is not relevant to the driving task. On a flat road, the driver will normally only see the sky and engine hood in these areas. An often-proposed use case for WSDs $[7,18]$ is the spatially registered (a.k.a. contactanalog) display of points of interest. These will also occupy the horizontally peripheral display areas, as POIs are mostly located in the horizontal plane around the car. 
In the current literature, the peripheral area is mostly considered inappropriate for information display. The best region for HUDs was found to be within the foveal FoV, slightly below the driver's line of sight $[9,24]$. Positioning information outside of this field, (i.e., with an eccentricity $>10^{\circ}$ ), is widely refused $[9,22,26]$ and there are two main arguments against the display in the periphery:

(1) The driver would have to move his or her head in order to perceive the displayed information [4]

(2) Outside of the foveal FoV, human perception is very limited, but also highly sensitive to light changes and movements. To such changes in the periphery, people often react in a knee-jerk manner by visually focusing on the stimulus. This sudden shift of the locus of attention is very safety-critical [16].

The first argument actually also covers standard displays, such as the instrument cluster or the central information display (CID) in the center stack. To make things worse, these standard displays are even much smaller, making them more difficult to read.

The second argument is relativized by the zoom-lens model of attention [5]. This model suggests that the field of view a person attentively observes can be reduced to increase the resolution at the focus point. Several studies found strong evidence that this model holds in driving situations: The response of drivers to stimuli presented in the periphery was tested in independent studies [3,14]. They all found, that the participants' performance in detecting peripheral stimuli depended on the demand of the driving situation. When the driving situation is highly demanding, the driver performs worse in detecting changes in the environment. In consequence, this also means that a demanding driving situation will automatically reduce the risk of looking to the side as a reflex to visual changes in the periphery. Drivers will only respond to peripheral information when the driving situation allows shifting their attention.

To complete these arguments, it is also important to consider the content and amount of information typically displayed on a WSD. All aforementioned studies use very basic stimuli, while the typical information on a WSD will most probably be more diverse and of lower importance. Peripheral information is the type of additional information, which is not central to the execution of a primary task. Such information can be provided to help a person do a better job or to keep track of less important tasks [15], but it is not asking for attention. In contrast, highly dynamic and demanding information would be very distracting and should not be displayed in the visual periphery. Also safetyrelevant information might be better placed in other areas, more central to the driver's locus of attention.

Finally, existing studies investigated the influence of the primary task on the peripheral task, but do not mention interactions. We could not find a study investigating the effect of visually attracting HUD/WSD content on driving.
In order to learn more about the potential risks and benefits of a peripheral display in the windshield area, we decided to perform a user study. 20 participants had to perceive and memorize information displayed in the middle area of the windshield (see figure 2) while performing lane changes. For this purpose, we built two experimental WSD setups, and since several focus distances have been suggested in the literature, we decided to investigate two alternative distances (see figure 1). We implemented two test setups, which can display information directly on the windshield as well as $1.7 \mathrm{~m}$ in front of the windshield. With these setups, we evaluated peripheral information regarding its memorability, measured impacts on driving performance and compared both WSD setups regarding user experience.

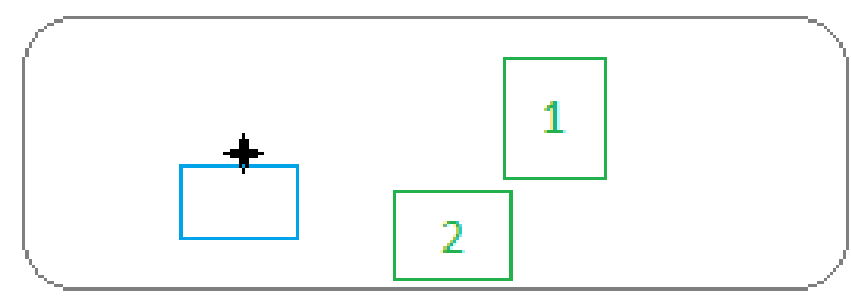

Figure 2: Area covered by a common HUD (blue) compared to the peripheral information areas in our user test (green) and the driver's average visual focus point (black cross)

\section{RELATED WORK}

Our work builds on existing studies of peripheral information display, as well as on other technical realizations of WSD test setups:

\section{Information Display in the Periphery}

Several studies found strong evidence that the zoom-lens model of attention holds in driving situations: Verwey presented digits for $750 \mathrm{~ms}$ in the periphery to drivers and found that the detection performance depends on the demand of the driving situation [23]. This effect seems to be related to a shrinking of the functional field of view [3]. The functional field of view represents the area in which a human can acquire information without moving the head or the eye. The size of this area is limited by visual resolution, but also depends on processing demands and even anxiety. This is supported by the zoom-lens model of attention.

In another study, Crundall et al. [3] asked participants to search for hazards in video clips showing different driving situations. As a secondary task, subjects had to respond with button presses to target lights, which were displayed in the periphery. They confirmed that driving situations with increased processing demand lead to a lower success rate for the peripheral targets. Moreover, lower success rates were also found for increased eccentricity. Isomura, Kamiya and Hamatani $[9,13]$ investigated a peripheral task at eccentricities between 10 and 40 degrees. A degradation of performance was found when the central task was either more demanding or the peripheral task was positioned more than 30 degrees off the central axis. 
Iino, Otsuka and Suzuki $[9,11]$ compared the reading times of information displayed on three HUDs positioned in the center, at $10^{\circ}$ eccentricity, and at $20^{\circ}$ eccentricity. For a speed below $70 \mathrm{~km} / \mathrm{h}$, the reading times did not vary. If the participants drove at a higher speed, they could read faster in the central display area. Between the HUDs at $10^{\circ}$ and $20^{\circ}$ eccentricity, no differences in reading time were found. The difference between central and peripheral HUDs was more prominent with higher speeds.

In summary, not only the humans' state and the demand of the central task, but also the display's eccentricity and the driving speed have an influence on the peripheral task. A speed below $70 \mathrm{~km} / \mathrm{h}$ allowed for the same performance in the entire area up to $20^{\circ}$ eccentricity. Independent of the speed, no difference was found between $10^{\circ}$ and $20^{\circ}$ eccentricity. However, more than $30^{\circ}$ seem to degrade the performance in a peripheral task. We therefore used an eccentricity of $30^{\circ}$ and a speed of $70 \mathrm{~km} / \mathrm{h}$ as the upper limits for our study design.

\section{Technological Realization}

A considerable number of HUD and WSD prototypes, test setups and concepts have been published by now. We reviewed the abilities, advantages and disadvantages of each approach in order to identify the ones, which could serve as a basis for our own test setup. In this review, we found the distance between the virtual image and the driver as a key differentiator. The proposed WSD systems provide information at various distances between $0 \mathrm{~m}$ and $50 \mathrm{~m}$ from the windshield $[20,25]$. We split the existing approaches according to their virtual image distance into the following three categories:

(1) Image presentation on the windshield

(2) Image presentation in front of the windshield

(3) Merging the virtual image and the real world

A large or even variable image distance, as needed for (3), is a desirable property of a WSD, but also increases its technical complexity. In order to keep the effort for our test setup manageable, we decided to focus on the categories (1) and (2) for this study. The following systems served as a basis for our own test setup:

\section{Image Presentation on the Windshield}

The display of information directly on the windshield constitutes the first step between standard in-vehicle displays, such as the instrument cluster or the central information display, and the classic Head-Up Display.

Image presentation on the windshield has been investigated by Volkswagen and General Motors [21,25]. Volkswagen already patented the integration of a transparent OLED into the windshield as well as the display of navigational hints on it. For this use case, they did neither try to cover the entire windshield area, nor to display the navigational arrows spatially close or adapted to the route. High-quality
OLEDs, which would facilitate such information presentation, are still very cost-intensive and can only be produced in small sizes. A simpler and less expensive approach is to use the windshield as a projection surface. General Motors [25] used a uni-colored laser projector to mark up traffic signs on the windshield. In order to ensure correct highlighting, this requires a fixed head position.

\section{Image Presentation in front of the Windshield}

Most prototypes and test setups, which display the image in front of the windshield, are based on the same principle: The distant display of the virtual image to the driver is realized by the reflection of a display or a projector image.

Also HUDs are technically based on this principle. A display, positioned perpendicular below a combiner mirror, serves as the image source. The combiner is either realized by an additional glass pane in front of the windshield or integrated into the windshield itself. It serves as a transparent mirror and projects the image of the display $2 \mathrm{~m}$ in front of the driver. Poitschke et al. [17] reflected the HUD image twice in order to reach a more distant image presentation. The proposed system is able to adapt to movements of the driver and thereby allows a correctly registered display of information.

Sato et al. [19] also used the described principle of reflection, but instead of positioning a display below the windshield, they attached a projector to the roof rack of a minivan and thereby created a street-ready prototype. A mirror and a retroreflector were used to project the image in front of the driver. The virtual image covers large parts of the windshield in front of the driver.

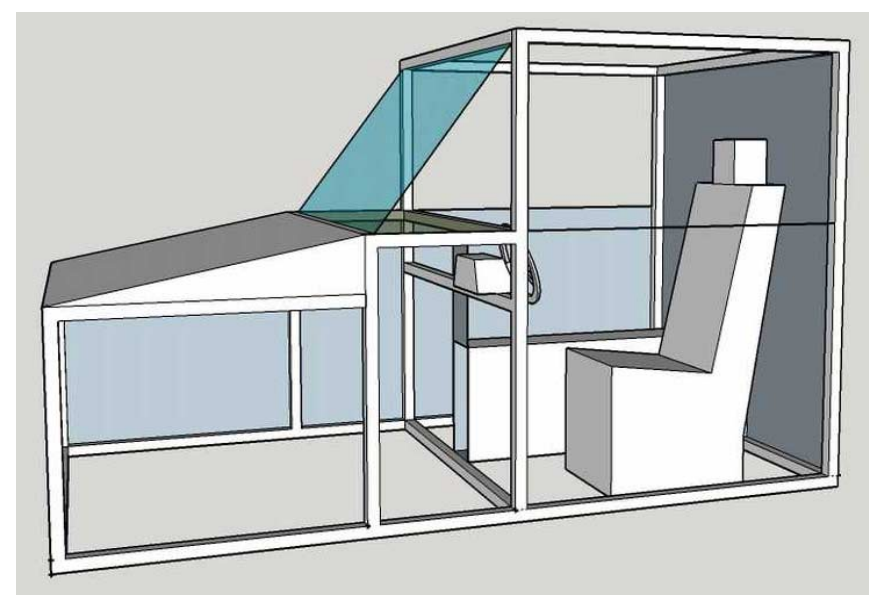

Figure 3: Basic construction of our test setup

\section{CONSTRUCTION OF THE TEST SETUPS}

The basic construction of the test setup is shown in figure 3 . It consists of a wooden frame disguised by fabric and is equipped with a real windshield, a real car seat, and a gaming steering wheel to control the openDS ${ }^{1}$ driving

\footnotetext{
${ }^{1}$ www.openDS.eu
} 
simulator. The simulator scene is projected on the wall, approximately $4 \mathrm{~m}$ in front of the test setup. The inside distances and angles are defined according to a BMW 1 series and, therefore, correspond to the dimensions of a real car. Our two test setups are implemented as follows:

\section{Image presentation on the Windshield}

As in the General Motors prototype, the first test setup is based on a high-end projector targeted onto a mirror, which is placed in the middle of the passenger compartment. The mirror forwards the image to the windshield, as visualized in figure 4. While direct projection leads to an irritating light refraction and glare and an insufficient imaging angle of the projector, the mirror enlarges the optical distance between the projector and the windshield and, therefore, scales the image to the windshield size. Depending on the driver's seat position, the distance between the driver and the image is approximately $0.7 \mathrm{~m}$.

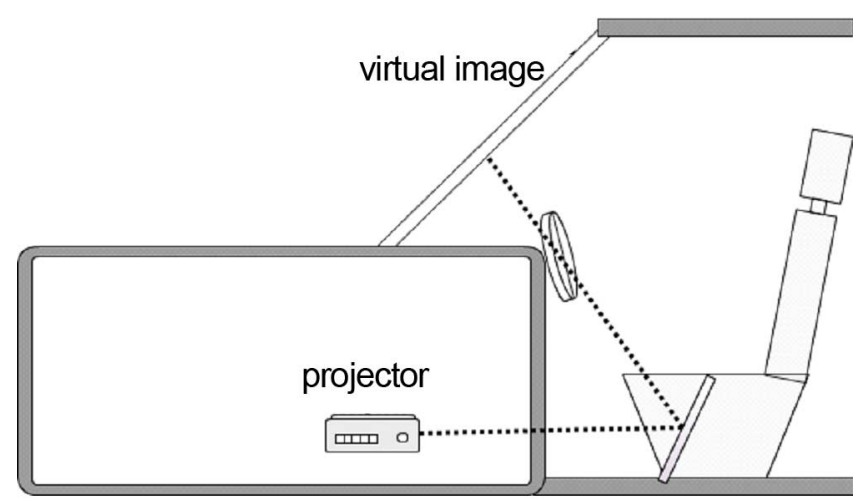

Figure 4: The projector image is forwarded to the windshield by a mirror.

As the windshield we used is highly transparent and does not diffuse enough light of the projector to provide a highquality image, we laminated it with a foil. After testing various types of foils, we found a simple gift wrap foil to be the best compromise. It is cheap and highly transparent, but at the same time provides a surprisingly good light refraction. Covering the windshield on the outer side, we obtained a bright, sharp and colorful image without any double image effects. However, the fringe of the virtual image was slightly distorted due to the curvature of the windshield. An example image provided by this test setup is shown in figure 7 . In the study, this setup will also be called the 'projector-based' approach.

\section{Image presentation in front of the Windshield}

In our second WSD test setup we lined up three displays below the windshield, which reflects the virtual image in front of the driver (see figure 5). We chose large displays (32 inch each) to create a virtual image that covers large parts of the view through the windshield. Even though we chose very bright displays, the room needed to be slightly darkened in order to obtain a crisp and colorful image.
The virtual image provided by this test setup is presented in figure 8 . For the study, we will refer to this as the 'screenbased' approach.

The virtual image distance depends on the position of the displays. When they are close to the floor, the virtual image distance is $1.7 \mathrm{~m}$ from the driver. At this distance, the image covers $60-70 \%$ of the driver's FoV. Lowering the distance between the display and the windshield decreases the virtual image distance, but also increases the subjective image size, as the closer image covers larger parts of the driver's field of view.

Currently, this WSD setup displays a slightly misaligned double image because both sides of the glass act as mirror surfaces. This double image cannot be corrected by adapting the image source. Instead, the windshield or combiner needs to be manipulated in form or material. One side of the combiner can be equipped with coatings which are anti-refractive or shaped like a wedge with increasing thickness [1]. As a first step, we decided to simply use a thinner acrylic pane to minimize the double image effect.

Compared with common HUDs, our distant image presentation does not require a fixed head position. The image is also visible to the driver when she or he moves or changes the seat position. Nevertheless, information, which is placed in relation and spatially close to environmental objects, has to be repositioned. Therefore, head-tracking will be needed in future iterations.

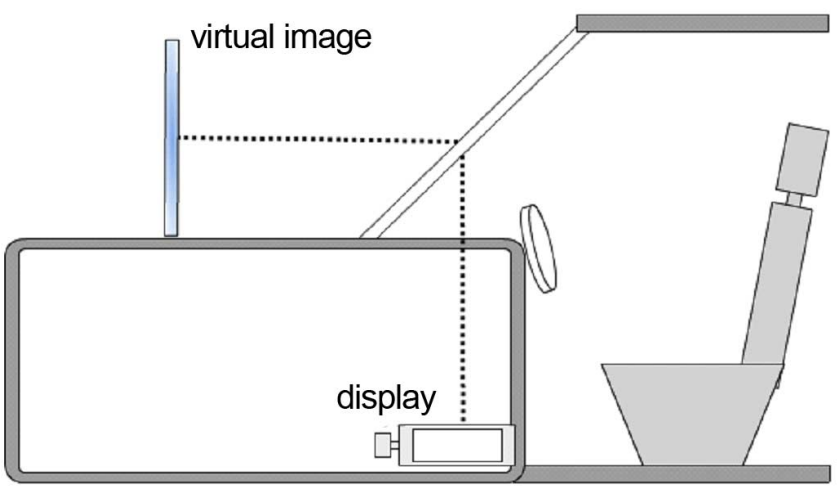

Figure 5: Three 32 inch displays are reflected in the windshield so that the virtual image appears in front of the driver.

For the study, we aimed to display one image in front of the car and the other one directly on the windshield, in order to compare these two viable and interesting approaches. Using the same hardware for both approaches would have been desirable, but was not possible due to the following reasons: It is not possible to use a projector for both images as a projected image can only be reflected to appear in front of the windshield if it first hits a projection surface on its way to the windshield. For this, it would need to be positioned at an angle or close to the windshield. The virtual image would then be of low contrast or positioned at a low image distance. This would decrease the difference between the two approaches. Neither was it possible to use a display for 
both approaches. For an image directly on the windshield, a transparent display, such as an OLED display would be needed. So far, no suitable display of this size is commercially available. Within the limits of the technologies we used, we made strong effort to provide comparable images in terms of brightness and resolution.

\section{USER STUDY}

In order to evaluate both windshield displays as well as the peripheral information displayed on them, we performed a user study with 20 participants. Subjects had to perceive and memorize information displayed on the WSD while performing lane changes. We investigated both systems regarding user experience and their impact on driving performance.

\section{Study Design}

In our study we aimed to investigate on the safety risk but also on the memorability of peripheral information, displayed on the two windshield display variants. Therefore, we decided to compare driving without an additional display (baseline) and driving with a windshield display (intervention). As we also wanted to compare both windshield display variants directly regarding user experience, we chose a within-subject study design. As each participant was supposed to test both WSD variants, for the memorability tests different peripheral information was provided. This resulted in a $2 \times 2$ study design.

\section{Participants}

We recruited 20 participants between 20 and 50 years of age (mean 28). Among them were six female and 14 male participants. All of them had a valid driver's license. For a counter-balanced study, we defined four test groups with different intervention configurations. We randomly assigned five out of 20 participants to the test groups B and C. Group A included six participants, group D four subjects. The defined groups A to D are depicted in table 1 .

Table 1: Test groups with assigned intervention configuration

\begin{tabular}{|c|c|c|c|}
\hline $\begin{array}{c}\text { Test } \\
\text { Group }\end{array}$ & $\begin{array}{c}\text { Intervention } \\
\text { Configuration }\end{array}$ & $\begin{array}{c}\text { Windshield } \\
\text { Display Variant }\end{array}$ & $\begin{array}{c}\text { Widget Board } \\
\text { Configuration }\end{array}$ \\
\hline \multirow{2}{*}{ A } & A & projector-based & 1 \\
\cline { 2 - 4 } & B & screen-based & 2 \\
\hline \multirow{2}{*}{ B } & A & screen-based & 1 \\
\cline { 2 - 4 } & B & projector-based & 2 \\
\hline \multirow{2}{*}{ C } & A & projector-based & 2 \\
\cline { 2 - 4 } & B & screen-based & 1 \\
\hline D & A & screen-based & 2 \\
\cline { 2 - 4 } & B & projector-based & 1 \\
\hline
\end{tabular}

\section{Lane Change Task}

A widely accepted approach to evaluate the effects of invehicle systems and applications on driving performance is the Lane Chance Task (LCT). This method demands the alteration of baseline and intervention phases, starting with a baseline. The lane change task prompts the driver every
$150 \mathrm{~m}$ to change to a specified lane and provides measures and methods for analyzing the driving performance [12]. The within-subject design required two intervention phases. We decided to schedule three baseline phases, in order to compensate learning effects. The last baseline gave time to the participants to disengage their minds before filling a comparative questionnaire. The overall procedure of the user study is depicted in figure 6 .

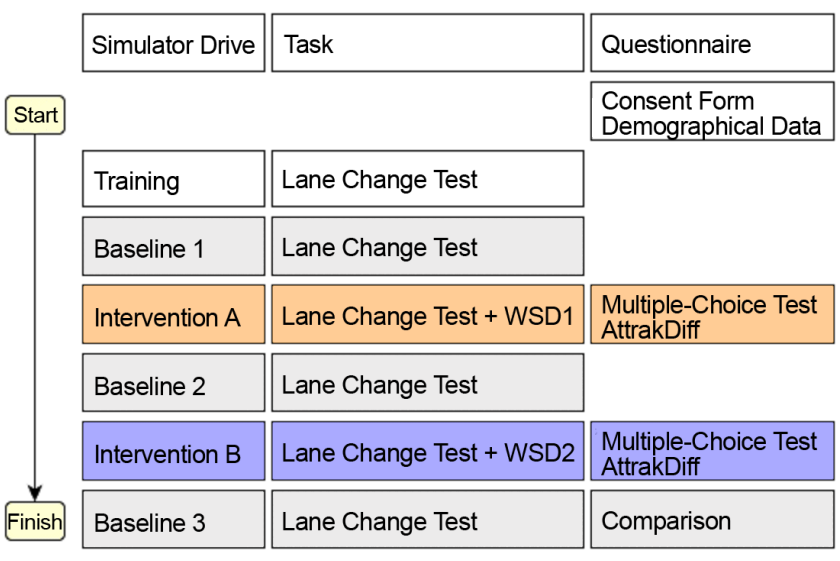

Figure 6: The procedure of the user study, including all tasks and questionnaires, took 35 to 45 minutes.

Each baseline and intervention phase was scheduled with three minutes, corresponding to three kilometers at a fixed speed of $60 \mathrm{~km} / \mathrm{h}$. In each phase, subjects had to perform 18 lane changes, spread evenly throughout time and length. The directions of the lane changes were randomized but equal for all subjects. The lane change instructions are presented as green spots, placed on overhead gantries.

\section{Memory Task}

As a secondary task, we chose a memory task. It guaranteed that participants would pay attention to the peripheral information but did not require any interaction. Therefore, participants were able to shift attention to the display whenever they wanted.

As an application for the secondary task, we chose a widget board. The widget board corresponds to the user's desire of being always online and getting information everywhere on time. Therefore, usually smartphones are used. Since handling the phone while driving is illegal in many countries, the display of smartphone apps on an in-vehicle display is also an attractive use case.

We implemented two widget boards with different applications. Providing not only different content but also different widgets was supposed to help participants not to confuse the information of both tests. The content was supposed to be homologous, of comparable complexity and of equal value in order to provide a comparable basis for measuring distraction and memorability. 


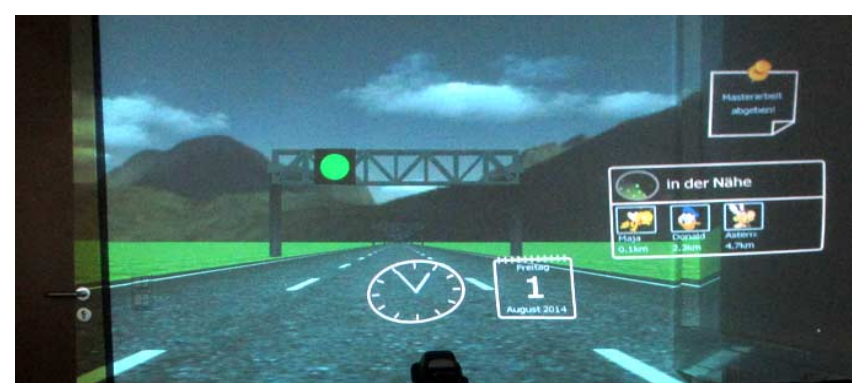

Figure 7: The first configuration of the widget board application displayed in front of the windshield.

The widget boards are located in the driver's peripheral field of view as recommended by Isomura $[9,13]$. We defined two regions for information presentation. Both regions were used in both widget boards. Information that is usually not accessible in vehicles, such as social media and weather information, was placed between the center and the right border of the WSD. This corresponds to an eccentricity of approximately $25-30^{\circ}$. The second cluster is positioned at the bottom in the middle of the windshield and presents information that is commonly visible on other displays. Apps such as the radio, clock or calendar are positioned at an eccentricity of approximately $15-20^{\circ}$. This arrangement was derived from common screen configurations and alignment of in-vehicle systems.

The position of the widgets was evaluated in pretests. It facilitated a good visual perception but also prevented the occlusion of important objects or parts of the driving scene. We also tested the amount of information displayed and the questions asked in the questionnaire before the actual user study started. Both were found to be manageable.

\section{Widget Board Configurations}

The first widget board configuration included the display of time, date, notifications, and friends nearby. In order to increase distraction and the amount of information to recall, both configurations included one widget which changed dynamically over time while driving. In this configuration it was the radar presenting friends nearby. The radar altered three times during one intervention: One friend appeared after 60 seconds, another one appeared after further 45 seconds and one disappeared after the next 45 seconds.

The second widget board configuration presented a radio, weather information, and Facebook status posts. In this widget board the Facebook post changed after 1.5 minutes, and a new post was displayed.

Both widget boards are depicted in the figures 7 and 8 . For technical reasons, both photos were taken from the middle of the test setup, not from the driver's point of view. Therefore, the widgets appear more central than they have actually been in the study.

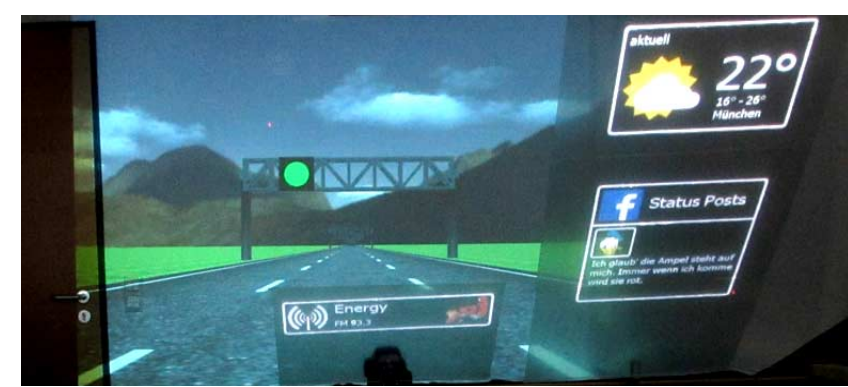

Figure 8: The second widget board configuration presented directly on the windshield.

\section{Data Collection}

We collected data about the users' experience and preference as well as the recall and driving performance:

\section{Questionnaires}

To test if the subjects paid attention to the peripheral information, we designed two recall questionnaires, one for each widget board configuration. Each questionnaire consisted of five questions about the content of the widgets. The question style was multiple-choice with four exactvalue answers, spread evenly across the range of possible answers. If text was involved, four possible answers containing some similarity, such as identical key words, were provided. This approach ensured the recall of the headline by context and not by recognition of a key word.

We assessed the users' feelings about both windshield display systems by two AttrakDiff questionnaires. The major advantage of this questionnaire is that it produces quantitative and comparative data. It consists of 28 items, expressing properties whose poles are defined by opposite adjectives. Items are measured on a seven-step scale and can be automatically decoded and analyzed on the AttrakDiff Website ${ }^{2}$.

Finally, participants had to provide their opinion about which system was more stunning or more readable. For this, we designed a questionnaire comparing the two WSD setups. Subjects were asked to fill out nine questions on a seven-step Likert-scale with the projector-based WSD on the one end and the screen-based WSD on the other. The position in the middle was neutral.

\section{Driving Performance Measurement}

To obtain objective data about the driving performance, the driving simulator logs the position of the vehicle. This allows the calculation of the mean deviation between optimal and driven line. The mean deviation is an indicator for driving quality and is sensitive to perception errors, reaction times, maneuvers, and lane keeping quality.

\footnotetext{
${ }^{2}$ www.attrakdiff.de
} 
The reaction time is measured as the time between the appearance of the lane change command and the driver's steering reaction. As the driver can be in the act of performing a correction movement when a new command appears, we set a minimum threshold of $300 \mathrm{~ms}$ for reliable recognition. As a last measure, we calculated the proportion of successfully performed lane changes. According to the LCT standard, the driver has to change to another lane (3500ms) and maintain stable control of the vehicle on this lane for at least $1500 \mathrm{~ms}$ to perform a lane change successfully.

\section{Procedure}

In our initial explanation, we introduced our goal of comparing two windshield displays. To support the participants' understanding, we explained the concept of a windshield display and the goal of comparing two systems to the participants. To support the explanation, we showed an example widget board and described the task of memorizing the displayed information. We also emphasized the precedence of good driving performance. After this briefing, participants were asked to fill out a short demographical data sheet and respond to a few questions about applications and widgets they use on their phone.

The driving test started with a training session. During this phase, subjects were allowed to drive around freely, practice and get familiar with both the steering wheel and the overall system of the test. Afterwards, participants had to perform the first baseline drive.

The test continued with the intervention drive A. 10 of 20 participants started the test with the image presentation on the windshield. The remaining 10 participants drove with the image presentation in front of the windshield first. Also the widget board configurations were provided counterbalanced in the first intervention. Afterwards, participants had to fill the recall and the AttrakDiff questionnaire. Subsequently, participants had to drive another baseline to clear and disengage their minds. During the intervention B subjects drove with the other windshield display and widget board configuration. Also after this drive, participants hat to fulfill a recall and an AttrakDiff questionnaire. Finally, subjects were asked to perform a last baseline drive. This baseline gave time to the participants to take one step back from the recently tested windshield display version. This was important for the comparative questionnaire, which was handed over to the participants at the end of the study.

\section{Results and Interpretation}

In the following we present the results of our user study. We will also point at and interpret interesting findings.

\section{Driving Performance}

As a measure of driving performance, we measured the mean deviation from optimal line, the reaction time, and the successfully performed lane changes. To analyze the difference between baseline and driving with the windshield displays, we always compared the intervention with the two surrounding baselines. As both widget boards were designed to display homologous content (comparable in complexity and of equal value), they were not differentiated in the driving performance evaluation.

The average values for mean deviation are depicted in table 2. Participants showed a marginally better performance in baseline driving compared with the two different WSD concepts. The screen-based WSD yielded slightly better results than its counterpart.

The exact values for reaction time are listed in table 3 and depicted in figure 9. An ANOVA test revealed, that the reaction times were significantly deteriorated by both WSD variants $(\mathrm{F}(3,19)=10.73, \mathrm{p}<.01)$. Reaction times are increased but still very good. At the speed of $60 \mathrm{~km} / \mathrm{h}$ the increase corresponds to a line segment of only $0.6 \mathrm{~m}$ and $0.8 \mathrm{~m}$ respectively. In the case of an emergency braking, the car would be stopped not even one meter further.

Table 2: Mean deviation increased only marginally when driving with a WSD.

\begin{tabular}{|l|c|c|c|}
\hline WSD Version & Baseline & Intervention & Effect \\
\hline Screen-based & $1.29 \mathrm{~m}^{2}$ & $1.32 \mathrm{~m}^{2}$ & $+1.9 \%$ \\
\hline Projector-based & $1.31 \mathrm{~m}^{2}$ & $1.33 \mathrm{~m}^{2}$ & $+1.6 \%$ \\
\hline
\end{tabular}

Table 3: Reaction time increased when driving with a WSD.

\begin{tabular}{|l|c|c|c|}
\hline WSD Version & Baseline & Intervention & Effect \\
\hline Screen-based & $729 \mathrm{~ms}$ & $763 \mathrm{~ms}$ & $+4,72 \%$ \\
\hline Projector-based & $737 \mathrm{~ms}$ & $784 \mathrm{~ms}$ & $+6,43 \%$ \\
\hline
\end{tabular}

The rate of successfully completed lane changes is depicted in figure 9. The success rates dropped from $95.7 \%$ to $90.3 \%$ when using the screen-based WSD and from $95.1 \%$ to $86.9 \%$ when using the projector-based system. An ANOVA showed, that this decline is statistically significant for the projector-based system $(\mathrm{F}(3,19)=6.38, \mathrm{p}<.01)$.

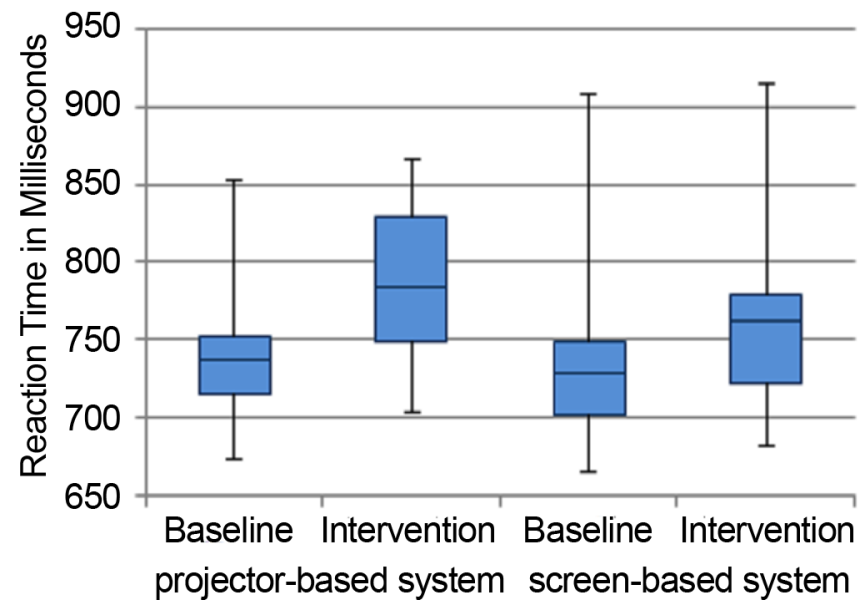

Figure 9: Reaction time increased when driving with a WSD, but especially when using the projector-based WSD 


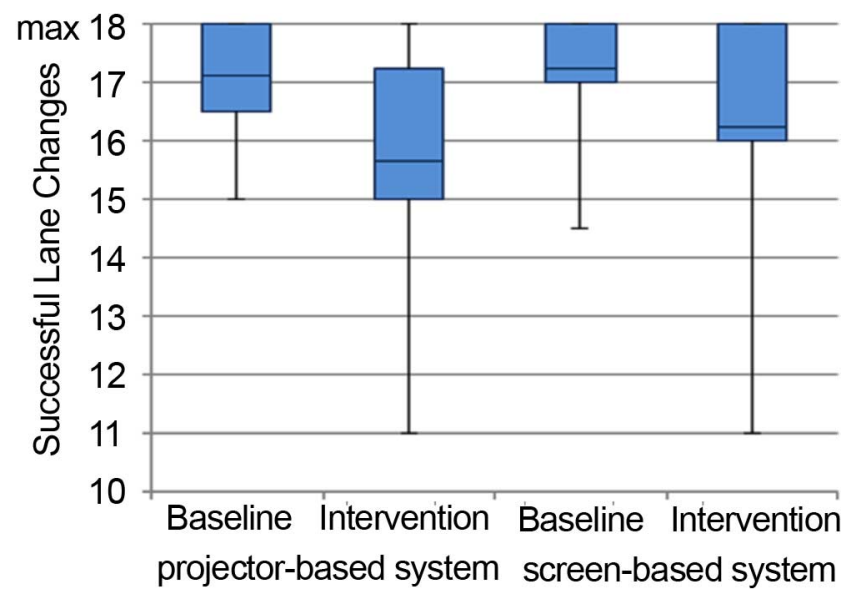

Figure 10: Lane change success rate was lowered when driving with both WSD variants.

Although we displayed the same information on both WSDs, we did not find the same effects on driving quality for the two versions. Both setups had only marginally different effects on the mean deviation, but looking at reaction time and success rate, the projector-based system had a considerably stronger effect than its counterpart.

Interpreting these results, the higher reaction times probably caused the drop in lane change success rate. The fact that success rate and reaction time are impaired, while mean deviation did not change noticeably, indicates that participants did not have problems to keep steady control of the vehicle but simply reacted more slowly.

\section{Recall Performance}

To verify whether subjects used the WSD application and were able to read and memorize the information displayed, multiple-choice questions were asked about the content that had been presented. In summary, participants performed very well on the recall questions (see figure 9). With $94 \%$ and $96 \%$ correct answers, both widget boards led to almost identical recall success rates. Eight correct answers were the minimum. Twelve of twenty users scored the maximum value of ten correct answers.

The high success rate in recalling the displayed information leads to the conclusion that participants used the windshield displays and looked at the peripheral information.

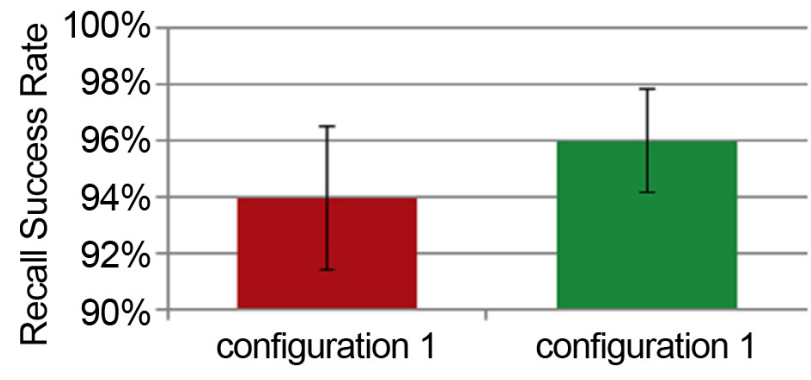

Figure 11: This chart shows the recall success rate and the error variances. The success rate of recalling the peripheral information was very high for both widget boards.

\section{User Experience}

The user experience questionnaire AttrakDiff was chosen to compare the two WSD types. In this questionnaire as well as in the additional, comparative questionnaire, we explicitly asked about the windshield displays, not about the widget board applications.

Figure 12 illustrates an overview of the user ratings. Both systems were rated as moderately attractive, but also show a clearly positive tendency. Both WSDs had good ratings for properties such as clearly structured, presentable, and innovative. These are all favorable aspects of a good user experience. They were also characterized as "good" and rated with equal values for this property.

For most properties, the screen-based setup received slightly better results. This system was valued as more presentable and manageable as well as clearer structured and less demanding. The projector-based system was rated remarkably better for the properties innovative and novel. This is most likely related to the prominence of HUDs and their distant image presentation. However, both systems need improvement, especially regarding professionalism and practicability. A system that is more human (natural) than technical in character and connects its users to the world rather than isolating them, would provide a more positive user experience.

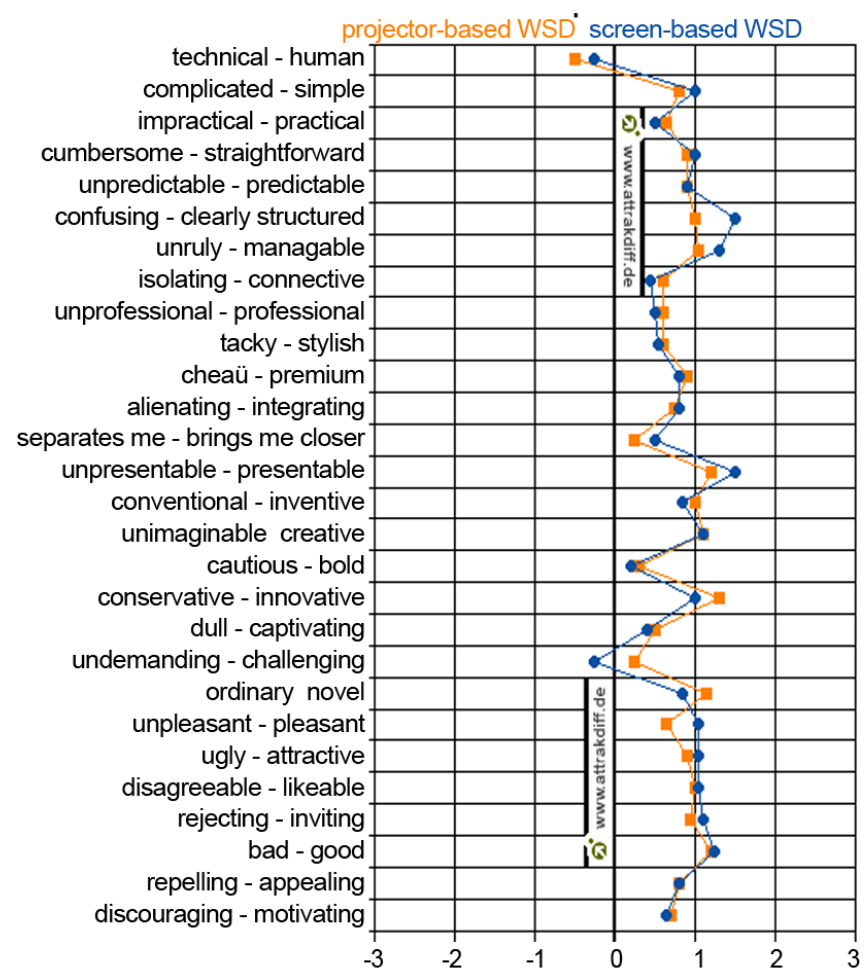

Figure 12: Both WSDs showed a good user experience.

The average ratings for pragmatic and hedonic quality of both systems correspond to a position within the neutral rectangle, but very close to the field 'desired' (see figure 13). The chart shows that the screen-based system has a slightly higher rating for pragmatic quality, corresponding 
to a rather task-oriented system. The projector-based system is rated with a marginally higher hedonic quality. This points to a rather self-orientated system. Though, both versions seem to provide a very good compromise of both orientations. The center points are surrounded by a small confidence rectangle, which indicates that subjects strongly agree in their evaluations.

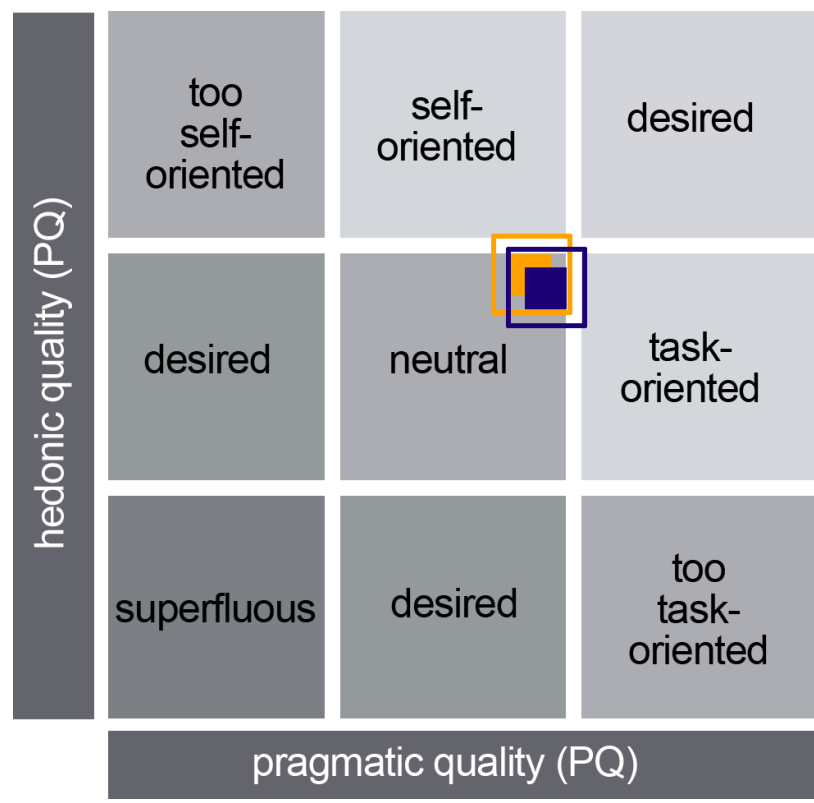

Figure 13: The pragmatic and hedonic quality of the screenbased (blue) and the projector-based (orange) WSDs are neutral but close to desired.

\section{User Preferences}

The last form of evaluation was a direct comparison of both WSD systems. Participants had to rate their preference on a scale from -3 to 3 with the projector-based system on the one end and the screen-based system on the other side. The value 0 represents a neutral attitude. A neutral rating means both systems are equal, but does not give an indication of how good or bad the systems are.

Figure 14 presents all user ratings. The average is presented by the group named 'complete' and the red line. Based on the ratings of the question about their favorite system, we differentiated three groups of users. Participants who valued this question with less than -1 , were assigned to the group preferring the projector-based WSD $(n=4)$. Subjects who rated the first question above +1 were assigned to the group in favor of the screen-based WSD $(n=10)$. Participants who valued this question in the range of -1 to +1 were assigned to the group 'neutral' $(\mathrm{n}=6)$. Three subjects assigned to the neutral group showed a tendency to the screen-based system and two tended to the projector-based system. One participant was completely neutral.

The preference for the screen-based system is not only demonstrated by the ratings of the first question. Looking at the overall ratings of the two groups favoring one system, it seems the group supporting the screen-based version was more enthusiastic. This group valued their preference with 1.42 on average. The group preferring the projector-based system only supported their preference with an average rating of 1.13 , which is close to the threshold to the neutral group. Looking at the overall ratings for all questions of all participants, the average value is 0.55 . All these values point at an overall preference for the image presentation in front of the display.

However, looking at the data in more detail, the screenbased system was not rated superior for all questions. Regarding distraction, both systems were rated equal (average $=-0.15$ ). Also the question which system made the subject feel safer was valued quite neutral (average $=0.45$ ). Both questions of course only measure perceived safety. The results indicate that participants perceived both systems as equally safe. The readability of text was rated equally good for both systems, but in general, information seems to be better recognizable when presented in front of the windshield. Especially graphics are rated easier to perceive on this WSD version.

Participants in favor of one system did not answer the questions about the system they could imagine to use and their preferred system equally. This indicates that either the need for a WSD is not big enough or that the image quality of both systems is not convincing enough to actually buy such system.

\section{DISCUSSION}

The widget boards were placed at an eccentricity of $15-30^{\circ}$ and the speed was fixed at $60 \mathrm{~km} / \mathrm{h}$. These values are below the thresholds found by $[11,13]$. Higher values would probably have led to a worse driving performance.

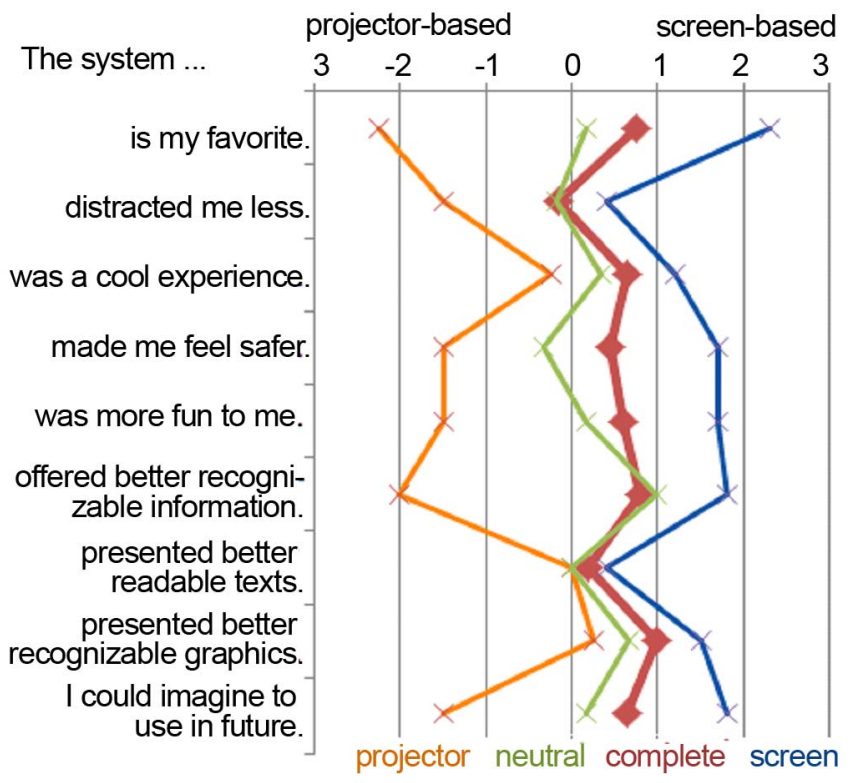

Figure 14: The comparison of both system shows that participants preferred the screen-based system (red line). 
To perceive information that was displayed on the widget boards, participants had to look to the side and to remove their focus from the driving scene. The fact that they actually looked at the widget boards is documented by a high recall success rate. Reviewing the results of our driving performance measurements, the required looks to the side had no remarkable influence on the mean deviation. However, we did find deteriorations in lane change success rate and reaction time, compared to baseline driving. As success rate depends on reaction time, these measures can be related to the same problem. These results indicate that participants simply reacted slower but did not have problems in keeping the vehicle steady on the road, when peripheral information was displayed.

The effects on driving performance were more prominent for the projector-based system than for its screen-based counterpart. The main difference of these systems is the virtual image distance. Consequently, it is most likely that the increase in reaction time is related to the image distance. This relationship has been pointed out in other literature as well [8]. When the distance between driver and image is increased, the distance between the driving scene and the virtual image is decreased simultaneously. The more distant image enables a faster accommodation and eventually leads to a better reaction time, compared to a virtual image closer to the viewer. This was already mentioned in other studies, on information presented in HUDs.

The user experience evaluation exposed that participants liked both windshield display variants. The AttrakDiff evaluation in figure 13 shows that the rectangles representing both systems are close to the field 'desired'. While not directly visible in the results of the AttrakDiff evaluation, the analysis of the comparative questionnaire showed that users preferred the screen-based version. Although subjects stated to like the windshield displays, they seem not to be too enthusiastic about using a system which is comparable to one of our test setups in the future. This fact shows that both versions need improvement. A major weakness seems to be the recognizability of information.

As evident from the figures 7 and 8 , both technologies provided homologous images. If at all, subjects may have had more problems to focus on and recognize the displaybased image (due to double-image and reflection) compared to the projected one. They may have rated the screen-based WSD better, if it had been without its flaws. Therefore, if results have been influenced by technology, we would expect this to be in favor of the projector-based WSD due to its slightly better image quality. Nevertheless, the screenbased WSD was rated better. The difference may therefore even be bigger without this potential bias.

\section{CONCLUSIONS AND FUTURE WORK}

Motivated by the potential risks and benefits of a peripheral display in the windshield area, we performed a user study.
We investigated the display of peripheral information at the height of the drivers' line of sight while driving. In related studies, the virtual image was either positioned within or close to the driver's central FoV or the displayed information was visually not demanding (only a simple stimulus) and investigated without observing its effects on driving performance. In our study, we set the lane change task as the primary, and a memory task as the secondary task. We investigated the peripheral information regarding its memorability as well as both WSD systems regarding user experience and preferences. Moreover, we measured impacts on driving performance. Interpreting the results of our study, we think that participants were able to look consciously at the peripheral information, presented on the screen-based windshield display, while keeping an acceptable level of driving performance.

For our user test, we built two windshield display test setups with different virtual image distances. The single test setups set themselves apart from the ones suggested in the literature by providing a large-scale image which also covers the outer parts of the windshield. This enables the evaluation of use cases, which refer not only to the road scene but also to the environment, such as the previously mentioned points of interest.

The distant image presentation is considered and was found to be the better approach but projection directly on the windshield is rather realizable. As follows, both visualization approaches should be further researched.

As a next step we want to enhance our test setups to reduce side effects related to image quality. Then we plan to test applications with a potential benefit for safety, and which rely on peripheral area. Such an application could for example be a hazard highlighting. People crossing from the sidewalk or wild animals in a rural environment could be marked up in the periphery using a windshield display.

Other future work, which we would like to encourage, would study peripheral information displayed more eccentric and also at larger distances. Moreover, we think that the human's response to a change of the peripheral information should be investigated more closely.

\section{ACKNOWLEDGMENTS}

This work was funded by IAV GmbH.

\section{REFERENCES}

[1] Betancur, J. A. (2011). Book chapter "Physical Variable Analysis Involved in Head-Up Display Systems Applied to Automobiles", Augmented Reality-Some Emerging Application Areas, edited by Dr. Andrew Yeh Ching Nee.

[2] BMW AG. BMW technology guide - BMW head-up display. http://www.bmw.com/com/en/insights/techno logy/technology_guide/articles/head_up_display.html, visited 28.11.2013. 
[3] Crundall, D., Underwood, G., \& Chapman, P. (1999). Driving experience and the functional field of view. Perception-London, 28(9), 1075-1088.

[4] Ecker, R. (2013). Der verteilte Fahrerinteraktionsraum (Doctoral dissertation, Ludwig-MaximiliansUniversität München), 34.

[5] Eriksen, C. W., \& Murphy, T. D. (1987). Movement of attentional focus across the visual field: A critical look at the evidence. Attention, Perception, \& Psychophysics, 42(3), 299-305.

[6] Foyle, D.C., Sanford, B.D. and McCann, R.S. (1991). Attentional issues in superimposed flight symbology. In R.S. Jensen (Ed.), Proceedings of the Sixth International Symposium on Aviation Psychology, 577-582.

[7] Fujimura, K., Xu, L., Tran, C., Bhandari, R., \& NgThow-Hing, V. (2013, October). Driver queries using wheel-constrained finger pointing and 3-D head-up display visual feedback. In Proceedings of the 5th International Conference on Automotive User Interfaces and Interactive Vehicular Applications , 5662.

[8] Gabbard, J. L., Fitch, G. M., \& Kim, H. (2014). Behind the Glass: Driver Challenges and Opportunities for AR Automotive Applications. Proceedings of the IEEE, 102(2), 124-136.

[9] Gish, K. W., \& Staplin, L. (1995). Human factors aspects of using head up displays in automobiles: A review of the literature.

[10] Horrey, W. J., Wickens, C. D., \& Alexander, A. L. (2003, October). The effects of head-up display clutter and in-vehicle display separation on concurrent driving performance. In Proceedings of the Human Factors and Ergonomics Society Annual Meeting, 47(16), 1880-1884.

[11] Iino, T., Otsuka, T., \& Suzuki, Y. (1988). Development of Heads-Up Display for a Motor Vehicle, 15-23.

[12] ISO, British Standards Institution. Road vehicles. Ergonomic aspects of transport information and control systems. Simulated lane change test to assess in-vehicle secondary task demand, 2010. ISO 26022.

[13] Isomura, A., Kamiya, K., \& Hamatani, K. (1993). Driver's Cognition in Peripheral Field of View.

[14] Jahn, G., Oehme, A., Krems, J. F., \& Gelau, C. (2005). Peripheral detection as a workload measure in driving: Effects of traffic complexity and route guidance system use in a driving study. Transportation Research Part F: Traffic Psychology and Behaviour, 8(3), 255-275.
[15] Maglio, P. P., \& Campbell, C. S. (2000, April). Tradeoffs in displaying peripheral information. In Proceedings of the SIGCHI conference on Human factors in computing systems (pp. 241-248). ACM.

[16] Poitschke, T. M. (2011). Blickbasierte MenschMaschine Interaktion im Automobil (Doctoral dissertation, Technische Universität München).

[17] Poitschke, T. M., Ablaßmeier, M., Rigoll, G., Bardins, S., Kohlbecher, S., \& Schneider, E. (2008, March). Contact-analog information representation in an automotive head-up display. In Proceedings of the 2008 symposium on Eye tracking research \& applications, 119-122.

[18] Rümelin, S., Marouane, C., \& Butz, A. (2013, October). Free-hand pointing for identification and interaction with distant objects. In Proceedings of the 5th International Conference on Automotive User Interfaces and Interactive Vehicular Applications (pp. 40-47). ACM.

[19] Sato, A., Kitahara, I., Kameda, Y., \& Ohta, Y. (2006). Visual navigation system on windshield head-up display. In Proc. 13th World Congress on Intelligent Transort Systems, CD-ROM.

[20] Takaki, Y., Urano, Y., Kashiwada, S., Ando, H., \& Nakamura, K. (2011). Super multi-view windshield display for long-distance image information presentation. Optics express, 19(2), 704-716.

[21] Transparente Anzeigevorrichtung für ein Kraftfahrzeug. Volkswagen AG, Patent, 2008.

[22] Tretten, P., Gärling, A., Nilsson, R., \& Larsson, T. C. (2011, September). An On-Road Study of Head-Up Display Preferred Location and Acceptance Levels. In Proceedings of the Human Factors and Ergonomics Society Annual Meeting, 55(1), 1914-1918.

[23] Verwey, W. B. (2000). On-line driver workload estimation. Effects of road situation and age on secondary task measures. Ergonomics, 43(2), 187-209.

[24] Weihrauch, M., Meloeny, G. G., \& Goesch, T. C. (1989). The first head up display introduced by general motors (No. 890288). SAE Technical Paper.

[25] Wu, W., Blaicher, F., Yang, J., Seder, T., \& Cui, D. (2009, October). A prototype of landmark-based car navigation using a full-windshield head-up display system. In Proceedings of the 2009 workshop on Ambient media computing, 21-28.

[26] Yoo, H., Tsimhoni, O., Watanabe, H., Green, P., \& Shah, R. (1999). Display of HUD warnings to drivers: determining an optimal location (No. UMTRI-99-9). 